\title{
Role of PLZF as a tumor suppressor in prostate cancer
}

\author{
Yang Jin ${ }^{1}$, Hatice Zeynep Nenseth ${ }^{2}$ and Fahri Saatcioglu ${ }^{1,2}$ \\ ${ }^{1}$ Institute for Cancer Genetics and Informatics, Oslo University Hospital, Oslo, Norway \\ ${ }^{2}$ Department of Biosciences, University of Oslo, Oslo, Norway \\ Correspondence to: Yang Jin, email: yangj@ibv.uio.no \\ Fahri Saatcioglu, email: fahris@ibv.uio.no \\ Keywords: promyelocytic leukemia zinc finger, prostate cancer, tumor suppressor, androgens
}

Received: March 16, 2017 Accepted: July 12, $2017 \quad$ Published: August 02, 2017

Copyright: Jin et al. This is an open-access article distributed under the terms of the Creative Commons Attribution License 3.0 (CC BY 3.0), which permits unrestricted use, distribution, and reproduction in any medium, provided the original author and source are credited.

\begin{abstract}
The promyelocytic leukemia zinc finger (PLZF), also known as ZBTB16 (Zinc Finger And BTB Domain Containing 16), is a transcription factor involved in the regulation of diverse biological processes, including cell proliferation, differentiation, organ development, stem cell maintenance and innate immune cell development. A number of recent studies have now implicated PLZF in cancer progression as a tumor suppressor. However, in certain cancer types, PLZF may function as an oncoprotein. Here, we summarize our current knowledge on the role of PLZF in various cancer types, in particular prostate cancer, including its deregulation, genomic alterations and potential functions in prostate cancer progression.
\end{abstract}

\section{INTRODUCTION}

The promyelocytic leukemia zinc finger protein (PLZF), also known as ZBTB16 (Zinc Finger and bric à brac, tramtrack, and broad (BTB) Domain Containing 16) or ZFP145 (Zinc Finger Protein 145), is a member of the Krüppel C2H2-type zinc-finger protein family and encodes a zinc finger transcription factor. PLZF was first identified in an acute promyelocytic leukemia (APL) patient with a $\mathrm{t}(11 ; 17)$ reciprocal chromosomal translocation which resulted in an in-frame fusion with the retinoic acid receptor alpha (RARA) gene. The PLZF-RAR fusion protein suppresses normal functions of PLZF and RARA, and is thus implicated in APL development. Since then significant new findings established PLZF as a multifaceted protein that regulates diverse biological processes, including cell proliferation, apoptosis, cell cycle, differentiation, and development (for a review, see [1]). Consistently, decreased PLZF expression has been associated with several types of cancer [2], in particular prostate cancer (PCa), which is the main focus of this review.

\section{Physiological and cellular functions of PLZF}

PLZF has a wide tissue distribution. Early studies focused on PLZF primarily in stem cells and early progenitor cells [3], but it is now established that PLZF is expressed in many cell types [4]. PLZF expression is higher in the brain, lung, endocrine and male-specific tissues including prostate and testis compared to other tissues (http://www.proteinatlas.org/) [4]. PLZF is predominantly nuclear in these tissues, except for the lung, in which PLZF expression is primarily cytoplasmic [5]. In the prostate, PLZF expression is largely restricted to the luminal cells, with expression weak/absent in basal or stromal cells $[5,6]$.

Previous studies showed that under normal physiological conditions PLZF is essential for normal differentiation and development. In 2000, a Plzf knockout mouse model was developed that displayed abnormal patterning of all limb skeletal structures [7]. Plzf ${ }^{-/}$mouse has major musculoskeletal-limb defects with homeotic transformations of vertebral segments, deformed cartilage and skeletal patterning, and alterations in digit formation. The abnormal skeletal phenotype resembles the general features of luxoid mouse, which was first described in the 1950s $[8,9]$. Genomic analysis revealed that the luxoid phenotype results from a frameshift mutation in the Plzf gene, which causes inhibition of progenitor cell apoptosis in the autopod region, thus blocking further limb development, possibly due to inhibition of Hox gene expression [9]. There are defects in germ cell production in both luxoid mutants and $\mathrm{Plzf}^{-/}$mice $[9,10]$, indicating a crucial role for Plzf in spermatogonial self-renewal. 
In germline progenitor cells, Plzf acts as a repressor of c-kit receptor expression to prevent spermatogenesis; in addition, it acts as an activator of the mTORC1 inhibitor Regulated in Development and DNA damage response 1 (REDD1) to prevent stem cell depletion [11].

PLZF has also been associated with stem and progenitor cell maintenance in hematopoietic stem cells (HSC) and neural progenitors. HSCs from luxoid mice exhibit an amplified aging phenotype associated with loss of stemness [12], while forced expression of PLZF immortalizes HSCs and myeloid progenitors [13]. PLZF is broadly expressed in earlier neural progenitors. Elevated PLZF expression sustains cells in a progenitor state and restricts neural differentiation by increasing FGFR3 expression and STAT3 activity [14]. PLZF maintains selfrenewal of stem cells in a cell-type specific manner and promotes stem cell differentiation, such as osteogenesis and chondrogenesis of mesenchymal stem cells. Recent findings implicated PLZF to play a critical role in the development of essentially all of the innate-like features of invariant Natural Killer T (NKT) cells [15-17].

PLZF mediates its effects mainly by modulating transcription, acting either as an activator or a repressor. PLZF consists of a BTB/POZ domain, a second repression domain (RD2), and a zinc finger domain (Figure 1). The BTB/POZ domain allows PLZF to form homodimers as well as heteromeric repressive complexes with $\mathrm{NCoR}$, SMRT and Sin3A [18, 19]. The zinc finger domain, composed of nine Krüppel-like $\mathrm{C} 2 \mathrm{H} 2$ zinc fingers, is responsible for DNA binding on target gene promoters [7]. PLZF activity is also regulated by post-translational modifications, such as acetylation [20-22], ubiquitination [23] and sumoylation [24] (Figure 1).

The mechanisms of PLZF-mediated repression of gene expression and the genes affected were recently reviewed in detail [1]. Briefly, PLZF recruits histone deacetylases (HDACs) and promotes the formation of the SMRT-mSin3-HDAC-NCoR corepressor complex to inhibit transcription $[18,19]$. In addition, PLZF can repress transcription by directly sequestering transcription factors. For example, PLZF binds the NF- $\kappa$ B p50 subunit and HDAC3 that assemble on the promoters of NF- $\mathrm{BB}$ target genes as transcriptionally inactive complexes $[21,22]$. This limits active NF- $\kappa$ B p50-p65 heterodimer formation, inhibits target gene expression, and thus leads to suppression of inflammatory responses [21, 22]. PLZF can also induce or suppress gene expression by directly binding to promoters of target genes through its Krüppellike $\mathrm{C} 2 \mathrm{H} 2$ zinc fingers [7]. So far, only a few genes have been shown to be direct PLZF targets [1]. As a BTB/ POZ domain containing protein, PLZF can also bind with Cullin 3 (CUL3) to form the E3 ubiquitin ligase complex, which occurs in the cytosol. For example, in Hela cells, PLZF-CUL3-ROC1 complex mediates the ubiquitination of ATG14L and thereby inhibits autophagy [25]. Thus PLZF has both nuclear and cytosolic functions in the cell.

\section{PLZF and cancer}

In early studies, PLZF was primarily linked to haematological malignancies, but it is now also implicated in various solid tumors (Table 1), including melanoma [26, 27], hepatocellular carcinoma [28], pancreatic cancer [29], colon cancer [30, 31], lung cancer [32], thyroid carcinoma [33], glioma [34] and PCa [5, 6, 35, 36].

PLZF expression is high in epidermal melanocytes from the foreskin, but is decreased in melanoma cell lines [26] and its expression is substantially lower in high-risk melanomas compared to low-risk ones [27]. Retroviral transduction of PLZF expression in melanoma cells resulted in a more differentiated, less malignant phenotype both in vitro and in vivo [26] which appears to be mediated by inhibition of the Pre-B-Cell Leukemia Homeobox 1 (PBX1) and Homeobox B7 (HOXB7) complex [37].

In human hepatocellular carcinoma (HCC), PLZF expression is significantly decreased at both mRNA and protein levels compared with paired adjacent normal tissue [28]. In addition, PLZF expression and several clinical features are positively correlated in HCC patients, such as the alkaline phosphatase levels and prothrombin time [28].

PLZF expression is reduced in pancreatic cancer relative to normal tissue, and is lost in 38 of 247 (15.4\%) cases [29]. Loss of, or reduction of, PLZF expression may be due to aberrant PLZF promoter methylation which was detected in $35.2 \%$ of pancreatic cancer cases (24).

PLZF expression is elevated in various colorectal cancer cell lines compared with isolated normal colonocytes [30]. It is inversely correlated to and transcriptionally suppressed by the expression of the CCAAT-DisplacementProtein (CUX1) [30]. Interestingly, a novel N-terminally truncated form of PLZF, and not the full length PLZF, is expressed in colorectal cancer cells [31]. The truncated PLZF lacks the N-terminal BTB/POZ domain and thus cannot translocate to the nucleus to inhibit transcription. In contrast to the tumor suppressive function of the intact PLZF protein, the truncated isoform appears to have a role in colorectal cancer cell adhesion and survival [31].

There is moderate to strong expression of PLZF in luminal cells of benign respiratory bronchus and welldifferentiated lung adenocarcinoma, but lower levels in the basal layer [5]. Furthermore, loss of PLZF expression is strongly correlated with increased grade, stage, lymph node metastasis and poor survival of lung squamous carcinoma and lung adenocarcinoma [5]. In pulmonary neuroendocrine tumors PLZF expression is negatively correlated to the tumor grade [38]. As in pancreatic cancer, loss of PLZF expression in non-small cell lung cancer may be due to promoter hypermethylation [39]. Interestingly, PLZF mainly localizes to the nucleus in the low-grade lung neuroendocrine tumor [40], while it is predominantly localized to the cytosol in the normal lung and lung adenocarcinoma [32]. 
PLZF is expressed in the normal thyroid and various thyroid lesions. Interestingly, whereas in the normal thyroid, adenomatous lesions, and follicular adenoma PLZF is mainly in the nucleus, in papillary thyroid carcinoma and anaplastic thyroid carcinoma it is primarily expressed in the cytosol (33). Furthermore, increased cytosolic PLZF expression was correlated with capsular invasion and lymph node metastasis in papillary thyroid carcinoma [33]. The basis for these intriguing findings on differential PLZF intracellular localization and correlation to thyroid pathologies is currently unknown.

The studies summarized above suggest that the fulllength nuclear PLZF may function as a tumor suppressor protein. On the other hand, there is evidence suggesting that in some cases PLZF may also serve as an oncoprotein. For example, IHC analysis showed a significant increase in the percentage of nuclear PLZF-positive cells in clear cell renal cell carcinoma, glioblastoma, and testicular seminoma tissues, compared with the corresponding normal tissues [34]. In addition, PLZF knockdown in clear cell renal cell carcinoma Caki-1 cells inhibited xenografted tumor growth in BALB/c-nu mice [34]. These data suggest that under certain circumstances PLZF may function as an oncoprotein.

It should be noted that many of the studies on PLZF so far utilized individual cell culture models in vitro, in particular while studying specific molecular interactions. Since the structural organization of living tissue composed of many cell types interacting with one another may affect these processes, further studies should focus on novel in vitro models, such as organoids and explant systems, but also in vivo models, e.g. utilizing the $\mathrm{Plzf}^{-/}$mice in the genetic background relevant to the cancer of interest.

\section{PLZF in prostate cancer}

\section{Androgen regulation of PLZF expression}

Androgen signaling is one of the most central pathways in PCa [41]. This is the basis for androgen deprivation therapies (ADT), including chemical/surgical castration, a mainstay treatment for metastatic PCa in the clinic. Although ADT is initially effective, most PCa patients become resistant and eventually develop castration resistant PCa (CRPC). PLZF expression was increased in castrated rat ventral prostate after androgen replacement [42]. This was further validated in androgen receptor (AR) positive PCa cell lines $\mathrm{LNCaP}[6,42]$, VCaP and 22Rv1 [35]. In addition, the antiandrogen bicalutamide inhibited PLZF expression in LNCaP cells [35]. Consistent with this, PLZF expression is undetectable in the AR deficient PCa cell lines DU145 and PC3 and ectopic expression of AR restored PLZF expression in DU145 cells [43], confirming androgen regulation of its expression, which is rapid and transient. These findings establish that PLZF is an androgen activated gene.

PLZF expression is also regulated by PTEN/AKT/ FOXO3 signaling, another important pathway in $\mathrm{PCa}$ cells [36]. There are seven putative consensus forkhead response element (FHRE) binding sites within the PLZF flanking sequences, including the $2.3 \mathrm{~kb}$ segment upstream of the transcription start site and the first exon. One site (FHRE1, +18 +24) that localizes within the first exon is essential for FOXO3 binding to the PLZF promoter [36]. FOXO3 knockdown in LNCaP cells inhibits PLZF expression while ectopic expression of constitutively active FOXO3 in PC3 PCa cells induces it. Consistently, PLZF, PTEN and FOXO3 protein expression are positively correlated in a cohort of human PCa specimens [36].

\section{PLZF in prostate cancer progression}

IHC analysis of multiple PCa cohorts indicated no significant change in PLZF expression in benign versus malignant prostate; furthermore, there was no correlation to Gleason score or T-stage [5]. Another IHC study found strong expression of PLZF in both benign prostate glands and low grade prostate tumors (Gleason pattern 3), but no significant difference between them [36]. However, in high

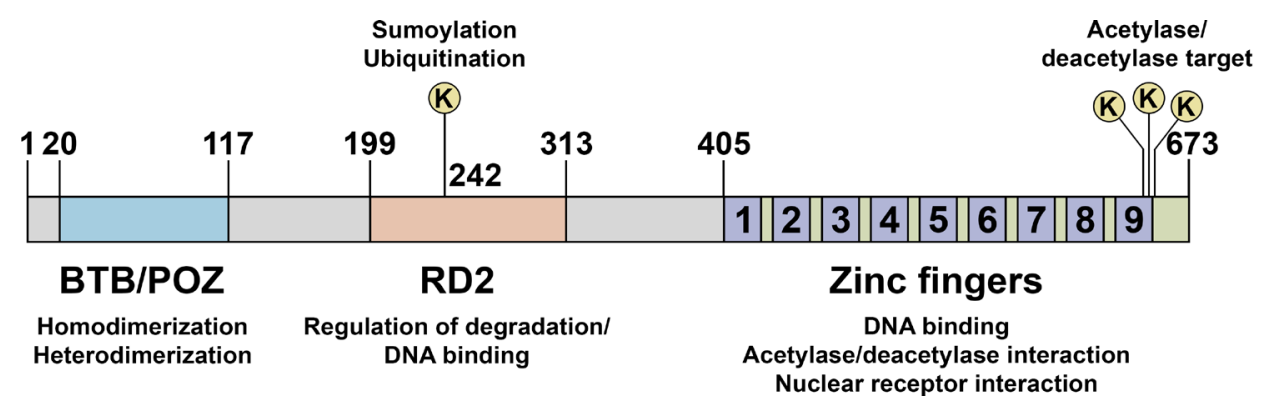

Figure 1: Schematic representation of PLZF functional domains. PLZF protein consists of a bric à brac, tramtrack, and broad/ poxvirus and zinc finger (BTB/POZ) domain, a second repression domain (RD2), and a zinc finger domain. The BTB/POZ domain allows PLZF to form homodimers as well as heterodimeric complexes with co-repressor molecules, such as N-CoR, SMRT and Sin3A. Lysine 242 in RD2 is a sumoylation and ubiquitination site. Sumoylation is critical for the transcriptional repression activity of PLZF whereas ubiquitination is involved in its degradation. The zinc finger domain, composed of nine Krüppel-like $\mathrm{C} 2 \mathrm{H} 2$ zinc fingers, is responsible for DNA binding on target gene promoters. Acetylation of lysine residues at the zinc finger domain enhances DNA binding and transcriptional repression by PLZF. 
Table 1: Alteration of PLZF in cancer

\begin{tabular}{|c|c|c|}
\hline Tumor type & Alterations & Ref. \\
\hline Clear cell renal cell carcinoma & Increased expression in tumor compared with normal tissue & {$[34]$} \\
\hline Colon cancer & $\begin{array}{l}\text { Increased expression in colorectal cancer cells compared with normal } \\
\text { colonocytes }\end{array}$ & {$[31,50]$} \\
\hline Glioblastoma & Increased expression in tumor compared with normal tissue & {$[34]$} \\
\hline Hepatocellular carcinoma & Decreased expression in tumor tissue compared to adjacent normal tissue & {$[28]$} \\
\hline Lung cancer & Decreased expression in high grade and high risk lung cancers & {$[32,38]$} \\
\hline Melanoma & $\begin{array}{l}\text { Decreased expression in high risk melanomas compared to low risk } \\
\text { melanomas }\end{array}$ & {$[26]$} \\
\hline Pancreatic cancer & $\begin{array}{l}\text { Down-regulation or loss of expression in tumor tissue compared to adjacent } \\
\text { normal tissue; aberrant promoter methylation }\end{array}$ & {$[29]$} \\
\hline Prostate cancer & $\begin{array}{l}\text { Down-regulation or loss of expression in high grade tumors; homozygous } \\
\text { deletion in CRPC }\end{array}$ & {$[5,6,35,36]$} \\
\hline Testicular seminoma & Increased expression in tumor compared with normal tissue & {$[34]$} \\
\hline Thyroid carcinoma & $\begin{array}{l}\text { Decreased nuclear expression and increased cytosolic expression in } \\
\text { papillary thyroid carcinoma tissue compared with normal thyroid tissue }\end{array}$ & {$[33]$} \\
\hline
\end{tabular}

grade PCa (Gleason pattern 4 and 5), PLZF expression was reduced/lost in $26 \%$ of the tumors and in up to $86 \%$ in metastatic specimens [5]. Another study also reported a decrease in PLZF expression in high grade PCa compared with low grade $\mathrm{PCa}$ [36]. In addition, PLZF expression was significantly lower in human bone metastatic CRPC tumor samples compared with hormone sensitive primary PCa tumors [35]. Whole-exome sequencing of metastatic CRPC specimens revealed that 5-7\% of tumors harbor PLZF homozygous deletions [35]. These findings suggest that PLZF may have a role in PCa progression.

\section{Tumor suppressive functions of PLZF in prostate cancer}

Several studies have assessed the possible role of PLZF in PCa. For example, ectopic expression of PLZF inhibits proliferation of PCa cell lines LNCaP [42], DU145 [43], and PC3 [36]. Conversely, inhibition of PLZF expression by siRNA or shRNA in LNCaP cells results in outgrowth $[6,35]$. Recent data suggest that PLZF knockdown promotes androgen-independent growth in LNCaP cells and it leads to resistance to the antiandrogen enzalutamide (32). Consistent with this, PLZF reexpression reverses androgen-independent growth (32). In addition, PLZF expression inhibited tumor formation by 22Rv1 cells (a CRPC model) under castrate levels of circulating androgens [35]. These results are consistent with the notion that PLZF is involved in CRPC development.

\section{Molecular mechanisms of PLZF action}

The data published so far suggest that PLZF acts as a tumor suppressor primarily via its ability to inhibit transcription. For example, the PLZF-RAR $\alpha$ fusion protein that arises in APL potently silences retinoic acid-regulated gene expression. Similarly, engineered PLZF fusion with estrogen receptor- $\alpha(\mathrm{ER} \alpha)$ or AR leads to inhibition of their target gene expression [44, 45]. In addition, PLZF can directly interact with retinoic acid receptor (RAR), $\mathrm{ER} \alpha$, glucocorticoid receptor (GR) and vitamin D receptor (VDR) and inhibit their transcriptional activity [46].

In LNCaP cells, PLZF knockdown significantly increases expression of AR target genes, whereas ectopic expression of PLZF suppresses androgen-regulated gene expression and inhibits AR transcriptional activity [6]. This appears to be due to inhibitory direct physical interactions between PLZF and AR in PCa cells, which result in suppression of $\mathrm{AR}$ transcriptional activity and inhibition of androgen dependent PCa cell growth [6].

As summarized above, PLZF expression in PCa cells is rapidly induced by androgens, while PLZF acts as a negative feedback regulator of AR. Interestingly, this feedback loop is inhibited by another androgen induced protein, Kallikrein Related Peptidase 4 (KLK4), whose expression is highly prostate enriched [6]. KLK4 expression is significantly increased in human PCa and blocking its expression inhibits proliferation and increases apoptosis [6]. Furthermore, siRNA targeting of KLK4 by systemic nanoliposomal delivery results in dramatic regression of xenografted PCa tumors in nude mice, establishing KLK4 as a key regulatory switch for PCa growth [6]. Mechanistic studies identified PLZF as an interacting partner for KLK4 that inhibits PLZF stability [6]. This relieves the inhibitory action of PLZF on androgen signaling in PCa cells resulting in tumor growth [6] (Figure 2).

Another pathway that is critical for proliferation and survival of PCa cells is PI3K-AKT-mTOR signaling $[47,48]$. A negative regulator of this pathway, the tumor suppressor protein PTEN, is mutated in the majority of 
PCa during progression. Earlier work in mouse germ-line progenitor cells has shown that PLZF induced expression of the mTOR inhibitor REDD1 [11]. PLZF is also required for REDD1 expression in PCa cells [6]. Consistent with this, chromatin immunoprecipitation analysis showed that PLZF bound to two distinct sites upstream of the human REDD1 promoter in an androgen dependent fashion [6] (see model in Figure 2). In summary, PLZF activates REDD1 expression and inhibits mTOR activation; KLK4 expression blocks PLZF-mediated REDD1 expression that results in activation of $\mathrm{mTOR}$ signaling, giving rise to tumor growth [6].

Global gene expression analysis in $\mathrm{PCa}$ cells revealed that PLZF-repressed genes are significantly enriched in the MAPK signaling pathway, including five genes with PLZF-binding sites in their regulatory regions: RRAS, MKNK2, DDIT3, JUND, and JUN (31).
In keeping with these data, PLZF knockdown induces phospho-ERK1/2 expression upon EGF stimulation in LNCaP cells [35] (Figure 2). In addition, PLZF inhibits expression of the homeodomain transcription factor PBX1 in androgen independent PCa cells [43]. Loss of PLZF releases PBX1 repression and may allow formation of a heterodimer with HOX, another homeodomain transcription factor. The PBX1-HOX protein complex may then in turn promote androgen-independent cell growth. These pathways and their known and putative interactions are schematically summarized in Figure 2.

\section{Summary and future perspectives}

PLZF is implicated in various cancer types as a tumor suppressor protein. In PCa, there is diminished or loss of PLZF expression in high grade tumors, especially in

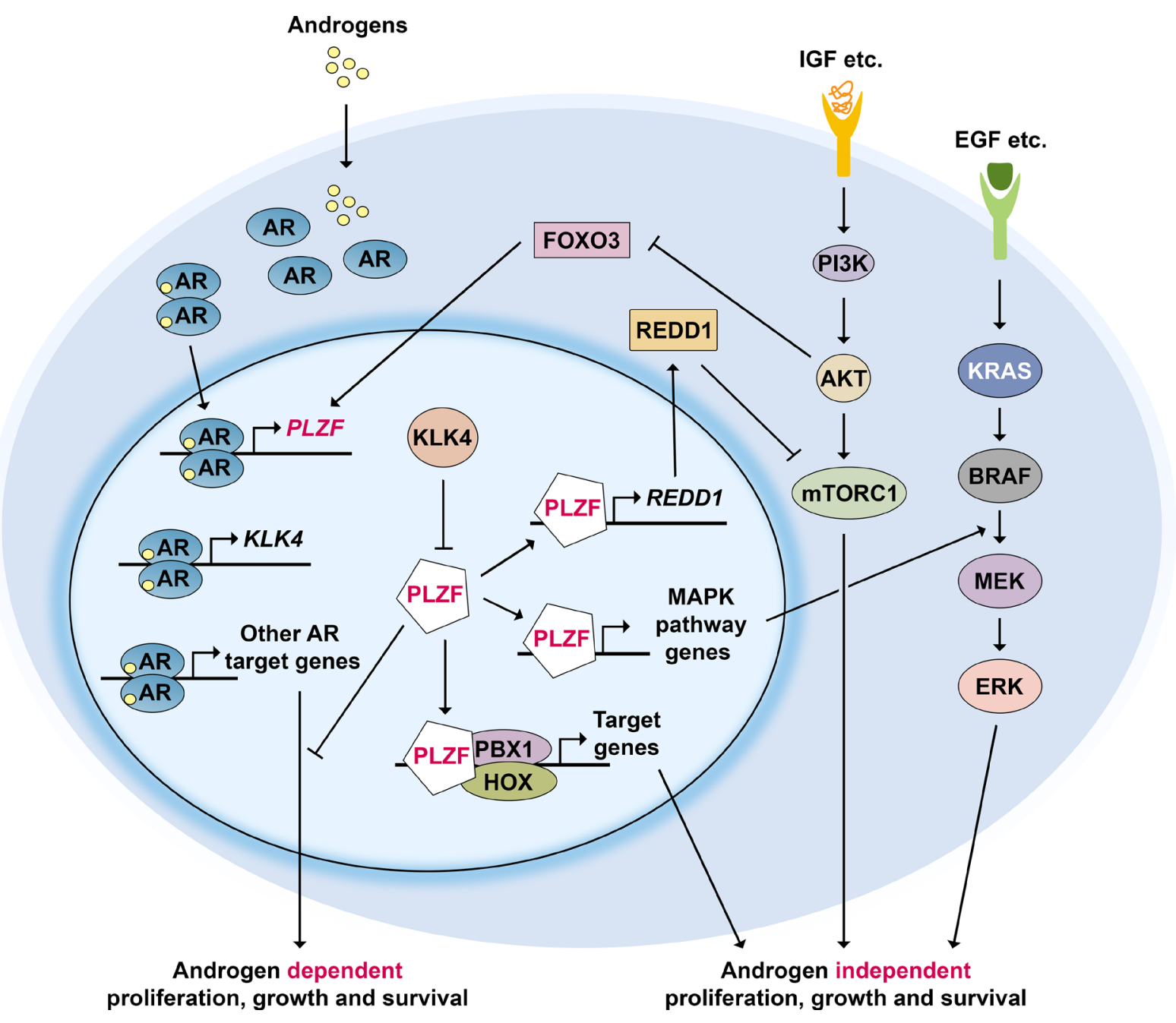

Figure 2: PLZF regulatory network in prostate cancer. Androgen receptor (AR) activation induces PLZF expression, while PLZF acts as a negative feedback regulator of AR. In addition, PLZF represses mTORC1 signaling by upregulating REDD1 expression and RAS/RAF/MEK signaling through increasing related gene expression. PLZF can also prevent the formation of PBX1-HOX heterodimers. Activation of PI3K-AKT pathway inhibits FOXO3 which normally activates PLZF expression. Highly prostate enriched protein KLK4 whose expression is androgen dependent, is a key regulator of PLZF: it physically interacts with and inhibits PLZF inhibited pathways, resulting in tumor growth. 
CRPC. PLZF inhibits PCa cell growth through inhibitory effects on AR, mTOR, and MAPK signaling (Figure 2). Thus, loss of PLZF leads to PCa cell growth, which is supported by in vitro and in vivo data. PLZF loss may also be involved in progression to CRPC. Therefore, reinstating PLZF expression/reactivation may be a novel strategy for PCa therapy. This could potentially be achieved by gene therapy of wild type PLZF, for example by adenovirus or oncolytic virus delivery, or by small molecules that interfere with inhibitory interactions, similar to that achieved for p53 [49]. In addition, given its interactions with several key signaling pathways in PCa cells, such as KLK4, understanding PLZF biology may help develop new biomarkers and therapeutic targets for PCa.

\section{ACKNOWLEDGMENTS AND FUNDING}

This work was supported by Norwegian Cancer Society grant 419204, Helse Sør-Øst grant 2014042, and Anders Jahre Fund grant 2016.

\section{CONFLICTS OF INTEREST}

None.

\section{REFERENCES}

1. Liu TM, Lee EH, Lim B, Shyh-Chang N. Concise Review: Balancing Stem Cell Self-Renewal and Differentiation with PLZF. Stem Cells. 2016; 34:277-287.

2. Suliman BA, Xu D, Williams BR. The promyelocytic leukemia zinc finger protein: two decades of molecular oncology. Front Oncol. 2012; 2:74.

3. Shaknovich R, Yeyati PL, Ivins S, Melnick A, Lempert C, Waxman S, Zelent A, Licht JD. The promyelocytic leukemia zinc finger protein affects myeloid cell growth, differentiation, and apoptosis. Mol Cell Biol. 1998; 18:5533-5545.

4. Uhlen M, Bjorling E, Agaton C, Szigyarto CA, Amini B, Andersen E, Andersson AC, Angelidou P, Asplund A, Asplund C, Berglund L, Bergstrom K, Brumer H, et al. A human protein atlas for normal and cancer tissues based on antibody proteomics. Mol Cell Proteomics. 2005; 4:1920-1932.

5. Xiao GQ, Unger P, Yang Q, Kinoshita Y, Singh K, McMahon L, Nastiuk K, Sha K, Krolewski J, Burstein D. Loss of PLZF expression in prostate cancer by immunohistochemistry correlates with tumor aggressiveness and metastasis. PLoS One. 2015; 10:e0121318.

6. Jin Y, Qu S, Tesikova M, Wang L, Kristian A, Maelandsmo GM, Kong H, Zhang T, Jeronimo C, Teixeira MR, Yuca E, Tekedereli I, Gorgulu K, et al. Molecular circuit involving KLK4 integrates androgen and mTOR signaling in prostate cancer. Proc Natl Acad Sci USA. 2013; 110:E2572-2581.

7. Barna M, Merghoub $T$, Costoya JA, Ruggero D, Branford M, Bergia A, Samori B, Pandolfi PP. Plzf mediates transcriptional repression of HoxD gene expression through chromatin remodeling. Dev Cell. 2002; 3:499-510.

8. Kienle M, Strong LC. Hematological studies of anemia in luxoid mice of a polydactylous descent: blood studies on mice of several strains. Blut. 1959; 5:335-343.

9. Buaas FW, Kirsh AL, Sharma M, McLean DJ, Morris JL, Griswold MD, de Rooij DG, Braun RE. Plzf is required in adult male germ cells for stem cell self-renewal. Nat Genet. 2004; 36:647-652.

10. Costoya JA, Hobbs RM, Barna M, Cattoretti G, Manova K, Sukhwani M, Orwig KE, Wolgemuth DJ, Pandolfi PP. Essential role of Plzf in maintenance of spermatogonial stem cells. Nat Genet. 2004; 36:653-659.

11. Hobbs RM, Seandel M, Falciatori I, Rafii S, Pandolfi PP. Plzf regulates germline progenitor self-renewal by opposing mTORC1. Cell. 2010; 142:468-479.

12. Vincent-Fabert C, Platet N, Vandevelde A, Poplineau M, Koubi M, Finetti P, Tiberi G, Imbert AM, Bertucci F, Duprez E. PLZF mutation alters mouse hematopoietic stem cell function and cell cycle progression. Blood. 2016; 127:1881-1885.

13. Ono R, Masuya M, Nakajima H, Enomoto Y, Miyata E, Nakamura A, Ishii S, Suzuki K, Shibata-Minoshima F, Katayama N, Kitamura T, Nosaka T. Plzf drives MLLfusion-mediated leukemogenesis specifically in long-term hematopoietic stem cells. Blood. 2013; 122:1271-1283.

14. Gaber ZB, Butler SJ, Novitch BG. PLZF regulates fibroblast growth factor responsiveness and maintenance of neural progenitors. PLoS Biol. 2013; 11:e1001676.

15. Beaulieu AM, Sant'Angelo DB. The BTB-ZF family of transcription factors: key regulators of lineage commitment and effector function development in the immune system. $\mathrm{J}$ Immunol. 2011; 187:2841-2847.

16. Kim EY, Lynch L, Brennan PJ, Cohen NR, Brenner MB. The transcriptional programs of iNKT cells. Semin Immunol. 2015; 27:26-32.

17. Savage AK, Constantinides MG, Han J, Picard D, Martin E, Li B, Lantz O, Bendelac A. The transcription factor PLZF directs the effector program of the NKT cell lineage. Immunity. 2008; 29:391-403.

18. Hong SH, David G, Wong CW, Dejean A, Privalsky ML. SMRT corepressor interacts with PLZF and with the PML-retinoic acid receptor alpha (RARalpha) and PLZFRARalpha oncoproteins associated with acute promyelocytic leukemia. Proc Natl Acad Sci USA. 1997; 94:9028-9033.

19. David G, Alland L, Hong SH, Wong CW, DePinho RA, Dejean A. Histone deacetylase associated with $\mathrm{mSin} 3 \mathrm{~A}$ mediates repression by the acute promyelocytic leukemiaassociated PLZF protein. Oncogene. 1998; 16:2549-2556.

20. Guidez F, Howell L, Isalan M, Cebrat M, Alani RM, Ivins S, Hormaeche I, McConnell MJ, Pierce S, Cole PA, Licht J, Zelent A. Histone acetyltransferase activity of p300 is required for transcriptional repression by the promyelocytic leukemia zinc finger protein. Mol Cell Biol. 2005; 25:5552-5566. 
21. Sadler AJ, Rossello FJ, Yu L, Deane JA, Yuan X, Wang D, Irving AT, Kaparakis-Liaskos M, Gantier MP, Ying H, Yim HC, Hartland EL, Notini AJ, et al. BTB-ZF transcriptional regulator PLZF modifies chromatin to restrain inflammatory signaling programs. Proc Natl Acad Sci USA. 2015; 112:1535-1540.

22. Sadler AJ, Suliman BA, Yu L, Yuan X, Wang D, Irving AT, Sarvestani ST, Banerjee A, Mansell AS, Liu JP, Gerondakis S, Williams BR, Xu D. The acetyltransferase HAT1 moderates the NF-kappaB response by regulating the transcription factor PLZF. Nat Commun. 2015; 6:6795.

23. Sobieszczuk DF, Poliakov A, Xu Q, Wilkinson DG. A feedback loop mediated by degradation of an inhibitor is required to initiate neuronal differentiation. Genes Dev. 2010; 24:206-218.

24. Kang SI, Chang WJ, Cho SG, Kim IY. Modification of promyelocytic leukemia zinc finger protein (PLZF) by SUMO-1 conjugation regulates its transcriptional repressor activity. J Biol Chem. 2003; 278:51479-51483.

25. Zhang T, Dong K, Liang W, Xu D, Xia H, Geng J, Najafov A, Liu M, Li Y, Han X, Xiao J, Jin Z, Peng T, et al. G-protein-coupled receptors regulate autophagy by ZBTB16-mediated ubiquitination and proteasomal degradation of Atg14L. Elife. 2015; 4:e06734.

26. Felicetti F, Bottero L, Felli N, Mattia G, Labbaye C, Alvino E, Peschle C, Colombo MP, Care A. Role of PLZF in melanoma progression. Oncogene. 2004; 23:4567-4576.

27. Brunner G, Reitz M, Schwipper V, Tilkorn H, Lippold A, Biess B, Suter L, Atzpodien J. Increased expression of the tumor suppressor PLZF is a continuous predictor of longterm survival in malignant melanoma patients. Cancer Biother Radiopharm. 2008; 23:451-459.

28. Hui AW, Lau HW, Cao CY, Zhou JW, Lai PB, Tsui SK. Downregulation of PLZF in human hepatocellular carcinoma and its clinical significance. Oncol Rep. 2015; 33:397-402.

29. Vincent A, Omura N, Hong SM, Jaffe A, Eshleman J, Goggins M. Genome-wide analysis of promoter methylation associated with gene expression profile in pancreatic adenocarcinoma. Clin Cancer Res. 2011; 17:4341-4354.

30. Frechette I, Darsigny M, Brochu-Gaudreau K, Jones C, Boudreau F. The Promyelocytic Leukemia Zinc Finger (PLZF ) gene is a novel transcriptional target of the CCAAT-Displacement-protein (CUX1) repressor. FEBS J. 2010; 277:4241-4253.

31. Jones C, St-Jean S, Frechette I, Bergeron D, Rivard N, Boudreau F. Identification of a novel promyelocytic leukemia zinc-finger isoform required for colorectal cancer cell growth and survival. Int J Cancer. 2013; 133:58-66.

32. Xiao GQ, Li F, Findeis-Hosey J, Hyrien O, Unger PD, Xiao L, Dunne R, Kim ES, Yang Q, McMahon L, Burstein DE. Down-regulation of cytoplasmic PLZF correlates with high tumor grade and tumor aggression in non-small cell lung carcinoma. Hum Pathol. 2015; 46:1607-1615.
33. Matsuzawa K, Izawa S, Ohkura T, Ohkura H, Ishiguro K, Yoshida A, Takiyama Y, Haneda M, Shigemasa C, Yamamoto K, Taniguchi S. Implication of intracellular localization of transcriptional repressor PLZF in thyroid neoplasms. BMC Endocr Disord. 2014; 14:52.

34. Choi WI, Kim MY, Jeon BN, Koh DI, Yun CO, Li Y, Lee CE, Oh J, Kim K, Hur MW. Role of promyelocytic leukemia zinc finger (PLZF) in cell proliferation and cyclindependent kinase inhibitor 1A (p21WAF/CDKN1A) gene repression. J Biol Chem. 2014; 289:18625-18640.

35. Hsieh CL, Botta G, Gao S, Li T, Van Allen EM, Treacy DJ, Cai C, He HH, Sweeney CJ, Brown M, Balk SP, Nelson PS, Garraway LA, et al. PLZF, a tumor suppressor genetically lost in metastatic castration-resistant prostate cancer, is a mediator of resistance to androgen deprivation therapy. Cancer Res. 2015; 75:1944-1948.

36. Cao J, Zhu S, Zhou W, Li J, Liu C, Xuan H, Yan J, Zheng L, Zhou L, Yu J, Chen G, Huang Y, Yu Z, et al. PLZF mediates the PTEN/AKT/FOXO3a signaling in suppression of prostate tumorigenesis. PLoS One. 2013; 8:e77922.

37. Shiraishi K, Yamasaki K, Nanba D, Inoue H, Hanakawa Y, Shirakata Y, Hashimoto K, Higashiyama S. Pre-Bcell leukemia transcription factor 1 is a major target of promyelocytic leukemia zinc-finger-mediated melanoma cell growth suppression. Oncogene. 2007; 26:339-348.

38. Hechtman JF, Beasley MB, Kinoshita Y, Ko HM, Hao K, Burstein DE. Promyelocytic leukemia zinc finger and histone H1.5 differentially stain low- and high-grade pulmonary neuroendocrine tumors: a pilot immunohistochemical study. Hum Pathol. 2013; 44:1400-1405.

39. Wang X, Wang L, Guo S, Bao Y, Ma Y, Yan F, Xu K, Xu Z, Jin L, Lu D, Xu J, Wang JC. Hypermethylation reduces expression of tumor-suppressor PLZF and regulates proliferation and apoptosis in non-small-cell lung cancers. FASEB J. 2013; 27:4194-4203.

40. Hechtman JF, Beasley MB, Kinoshita Y, Ko HM, Hao K, Burstein DE. Promyelocytic leukemia zinc finger and histone H1.5 differentially stain low- and high-grade pulmonary neuroendocrine tumors: a pilot immunohistochemical study. Human pathology. 2013; 44:1400-1405.

41. Heinlein CA, Chang C. Androgen receptor in prostate cancer. Endocr Rev. 2004; 25:276-308.

42. Jiang F, Wang Z. Identification and characterization of PLZF as a prostatic androgen-responsive gene. Prostate. 2004; 59:426-435.

43. Kikugawa $T$, Kinugasa $Y$, Shiraishi K, Nanba D, Nakashiro K, Tanji N, Yokoyama M, Higashiyama S. PLZF regulates $\mathrm{Pbx} 1$ transcription and $\mathrm{Pbx} 1-\mathrm{HoxC} 8$ complex leads to androgen-independent prostate cancer proliferation. Prostate. 2006; 66:1092-1099.

44. Buluwela L, Pike J, Mazhar D, Kamalati T, Hart SM, AlJehani R, Yahaya H, Patel N, Sarwar N, Heathcote DA, Schwickerath O, Phoenix F, Hill R, et al. Inhibiting estrogen 
responses in breast cancer cells using a fusion protein encoding estrogen receptor-alpha and the transcriptional repressor PLZF. Gene Ther. 2005; 12:452-460.

45. Pike J, Holmes D, Kamalati T, Davies D, Tolhurst R, Mazhar D, Fishpool S, al-Jehani R, Waxman J, Zelent A, Lemoine NR, Ali S, Buluwela L. Silencing of androgenregulated genes using a fusion of AR with the PLZF transcriptional repressor. Oncogene. 2004; 23:7561-7570.

46. Martin PJ, Delmotte MH, Formstecher P, Lefebvre P. $\mathrm{PLZF}$ is a negative regulator of retinoic acid receptor transcriptional activity. Nucl Recept. 2003; 1:6.

47. Taylor BS, Schultz N, Hieronymus H, Gopalan A, Xiao Y, Carver BS, Arora VK, Kaushik P, Cerami E, Reva B,
Antipin Y, Mitsiades N, Landers T, et al. Integrative genomic profiling of human prostate cancer. Cancer Cell. 2010; 18:11-22.

48. Roychowdhury S, Chinnaiyan AM. Advancing precision medicine for prostate cancer through genomics. J Clin Oncol. 2013; 31:1866-1873.

49. Lane DP, Cheok CF, Lain S. p53-based cancer therapy. Cold Spring Harb Perspect Biol. 2010; 2:a001222.

50. Mariani F, Sena P, Magnani G, Mancini S, Palumbo C, Ponz de Leon M, Roncucci L. PLZF expression during colorectal cancer development and in normal colorectal mucosa according to body size, as marker of colorectal cancer risk. ScientificWorldJournal. 2013; 2013:630869. 